\title{
Proceeding
}

Supplementary Issue: Summer Conferences of Sports Science. $8^{\text {th }}$ International Workshop and Conference of the International Society of Performance Analysis of Sport (ISPAS), 11-1 th of September 2019 (Budapest, Hungary) "Technology meets Practice and Science".

\section{Optimization techniques for basketball players under the convex risk measures}

\author{
LAZAROS NTASIS \\ Department of Economics, University of Peloponnese, Tripoli, Greece
}

\begin{abstract}
The effect of playing "home" or "away" and many other determinants, such as shooting percentage, the offensive rebounds, the turnovers and the number of free throws, have been hypothesized as influencing the outcome of major basketball matches. The optimal selection by team coaches during the game is the main unsolved problem. Due to their axiomatic foundation and properties, the convex measures are becoming a powerful tool in performance analysis. In this paper, we will review the fundamental structural concepts of convex performance optimization within the framework of convex analysis. We investigated the ARC optimization program over the coach selection and results found that convex optimization problem fitted optimal under the different game circumstances. Keywords: Convex set; Optimization; Basketball; Performance indicators.
\end{abstract}

\section{Cite this article as:}

Ntasis, L. (2019). Optimization techniques for basketball players under the convex risk measures. Journal of Human Sport and Exercise, 14(5proc), S2435-S2440. doi:https://doi.org/10.14198/ihse.2019.14.Proc5.59

\footnotetext{
Corresponding author. Department of Economics, University of Peloponnese, Tripoli, Greece.

E-mail: Intasis@uop.gr

Supplementary Issue: Summer Conferences of Sports Science. $8^{\text {th }}$ International Workshop and Conference of the International Society of Performance Analysis of Sport (ISPAS), 11-13 th of September 2019 (Budapest, Hungary).

JOURNAL OF HUMAN SPORT \& EXERCISE ISSN 1988-5202

(c) Faculty of Education. University of Alicante

doi:10.14198/jhse.2019.14.Proc5.59
} 


\section{INTRODUCTION}

The study of basketball games as competition has been used to identify variables that can distinguish between successful teams and players. Indeed, this field of research has recently become a subject of practical and scientific interest to coaches and sports scientists. The core coaches' problems are how they can select the optimal player during the game. Furthermore, performance indicators are used to assess the performance of an individual, a team or elements of a team (Csataljay, 2017). Due to frequent appearance in the real world, solving constrained optimization problems, especially nonlinear optimization problems, are of scientists' great interests in recent decades. Structural optimization and convex optimization problems are just a few fields in which constrained optimization problems are met. There are several deterministic algorithms which are efficient to solve constraint optimization problems, such as recursive quadratic programming, projection method and the generalized reduced gradient method (Luenbergen, 1984). The research interest due to recent decades is how to solve real word optimization problems in many fields such as business, industry, engineering, sports etc. The Athletes-Return-Correction (ARC) is a novel algorithm designed based on players optimal decision by coaches. The (ARC) program optimizes the selection of the basketball players at the next game based on time varying dataset and performance characteristics of each player. In addition, ARC program is a research-based on optimization problem techniques under the convex risk measures introduced by Artzner et al. (1999) approach that attempts to improve the optimal decision in real-time. Well-chosen performance indicators help the coach to identify good and bad performances (Bartlett, 2001; Hughes and Franks 1997, 2004; 2008), either at an individual or team level. Performance indicators are often used to define the differences between the players. We used players statistics to identify to the optimal weight of the linear players return formula. Additionally, other researchers (Mendes and Janeira, 2001; Tsamourtzis et al., 2002) found that defensive rebounding is the main factor that distinguishes winning and losing teams in basketball. According to Sampaio and Janeira (2003) investigated that game location (home and away games) and game type (regular season or playoff) effects game results. More recently, Oliver (2004), investigated four factors may be determinant to win basketball games, the shooting percentage from the field, the offensive rebounds, the turnovers and the number of free-throw attempts.

Under stressful basketball games, the coach has a big role and responsibility in the formation of team tactics. Results of analysing close games give useful information about the most important elements that distinguish winning and losing teams. Knowing the crucial performance indicators of close games allows coaches to prepare more detailed practice and game plans to build up the best winning strategy. The main purpose of the current study is to find the optimal strategy under the convex measures based on the returns of each player at a different type of matches of the NBA 2019 Champion.

The present paper is organized as follows. The next section introduces and describes the methodology and the steps to be followed. In Section 3 the case of optimization techniques evaluation is introduced, and the application of the proposed method is explained, and results presented. Finally, in Section 4 we discuss the results and main advantages of the proposed method.

\section{METHOD}

The optimization process typically begins with the construction of a linear model that is solved based on the literature determinants of Oliver (2004). Data processing was made by R i386 3.5.0 statistic package and the game analysis was used to classify the matches into three types such as close games with final score differences between 1 and 9 points, balanced games (10-19 points) and unbalanced games (20 and more 
points difference). Finally used observations are 288 collected from basketball reference database including Toronto Raptors 2019 play-off results.

We investigated a linear model to present the return of the basketball player in time t during the gam

$$
\begin{gathered}
r(x)_{t}=w_{1} \text { assist }+w_{2} \text { twopoint }+w_{3} \text { threepoint }+w_{4} \text { freethrows }+w_{5} \text { points } \\
+w_{6} \text { rebounds }+w_{7} \text { minutesongame }
\end{gathered}
$$

Where $w_{1}$ is the sum of correct and wrong passes, two-point shoot is the percentage of correct shoots, $w_{3}$ is the percentage of three points shoots, $w_{4}$ is the percentage of free throws, $w_{5}$ is the sum of points, $w_{6}$ is the total rebounds per minute and $w_{7}$ are the minutes where the player gives higher return than the other player in the same position. The defined player's positions are five; point guard, shooting guard, small forward, power forward and center. The total weights of each determinant change if the position of the players is in group j includes (point guard, shooting guard, small forward) or in the group, $\mathbf{q}$ includes (power forward and center)

Optimizing the following dynamic problem gives the return of each player:

Maximize

$w_{i} \in[0,1]$

$$
\text { subject to } \quad \sum_{i=1}^{N} w_{i}=1
$$

Where $\mathbf{x}$ is vector of players $\left(x_{1}, \ldots, x_{n}\right)$ and $w_{i}$ are the weights of each determinant. The evaluation of $r(x)$ investigated from the correlation $\sigma_{\rho}$ between the best player of the team into each position and the difference between player return $r(x)_{i}$ and higher return player into the season $r(x)_{j}$.

$$
R_{x}=\frac{\left(r(x)_{i}-r(x)_{j}\right)}{\sigma_{\rho}}
$$

Definition 2.1 Convex Measures [1] A mapping $\rho: X \rightarrow R$ is called a convex measure, if and only if

Convex: $\rho(\lambda X+(1-\lambda) Y) \leq \lambda \rho(X)+(1-\lambda) \rho(Y)$ for $\lambda \in[0,1]$.

Monotone: $X \leq Y$ then $\rho(X) \geq \rho(Y)$, where $X \leq Y \Leftrightarrow X(\omega) \leq Y(\omega)$ for all $\omega \in \Omega$

Translation invariant: if a is constant then $\rho(a \mathbf{1}+X)=-a+\rho(X)$

Adding to these properties positive homogeneity one obtains:

A convex risk measure $\rho$ is called coherent

if in addition:

Positive homogeneity: if $\lambda \geq 0$ then $\rho(\lambda \cdot X)=\lambda \cdot \rho(X)$

Furthermore, we minimize the following linear problem selected the best strategic based on convexity theorem: 


$$
\begin{aligned}
& \min _{j, q} \rho_{\pi c}(r(x)) \\
& \text { subject to } \quad E[r(x)] \geq u \\
& \sum_{i=1}^{N} x_{i}+x_{N+1}+\sum_{i=1}^{N} q_{i}=W \\
& x_{i} \geq 0, i=1, \ldots, N+1
\end{aligned}
$$

Were $r(x)$ is the return calculated in equation (1.1) and $\rho_{\pi c}$ is the convex risk measures. Furthermore equation (1.6) take into consideration only returns betters than the return of a player who plays currently. One of the core characteristics of convex measures is that they can present as a cone.

Let $\rho(x)$ be a cone. The set

$$
\rho(\mathrm{x})^{*}=\left\{x_{i} \mid x^{T} \geq 0 \text { for all } x \in \rho(\mathrm{x})\right\}
$$

is called the dual cone of $\rho(x)$. As the name suggests, $\rho(x)^{*}$ is a cone, and is always convex, even when the original cone $\rho(\mathrm{x})$. Geometrically, $x_{i} \in \rho(\mathrm{x}) *$ if and only if $-x_{i}$ is the normal of a hyperplane that supports $\rho(x)$ at the origin. This is illustrated in Figure 1.

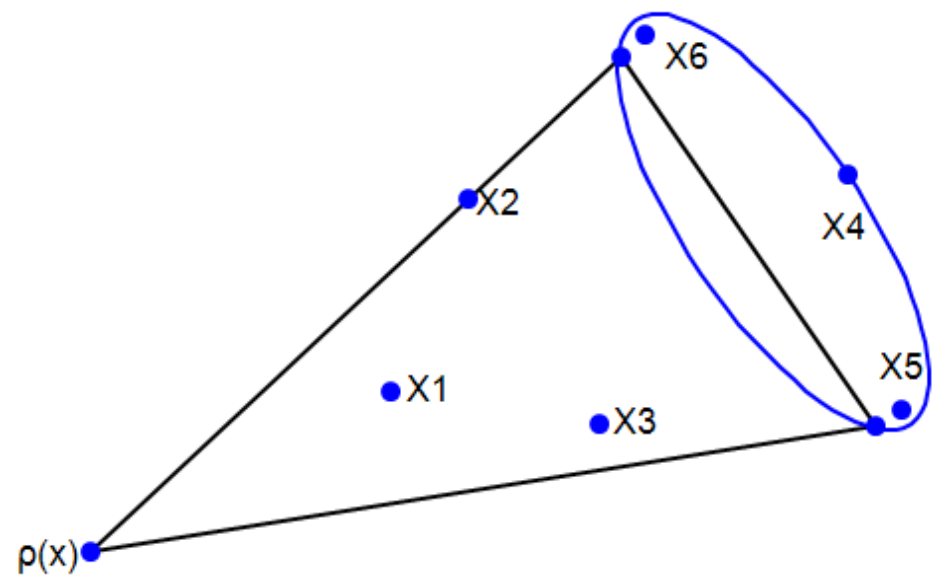

Figure 1. The cone $\rho(x)$ with error observations and accepted observations.

The players $X_{1}, X_{2}, X_{3}$ are in the accepted. On the other hand, players $X_{3}, X_{4}, X_{5}$ are in the rejected area, this area represented as penalty area out of the cone.

\section{RESULTS AND DISCUSSION}

The aim of the paper was to prove how the ARC choosing the best possible strategy in every game. Thus, in this section, we show an example of how our algorithm works for every possible target is known. Based on playoff games of Toronto Raptors basketball teams. We presented in Table 1.1 the results of coach strategy results about final score differences between ( 1 and 9 points), balanced games (10-19 points) and unbalanced games (20 and more points difference).

Coaches weight percentage calculated by the time and points of players during the game. In addition, the weight percentage of each player solved by minimizing theorem (1.4), on the other hand, coach optimal 
weight multiple with returns calculated from (1.1) equation. Figure 2 presents a time series and the results of all players multiplied by the optimal weight in two different decisions.

Table 1. Players average Points and Minutes per Game.

\begin{tabular}{|c|c|c|c|c|c|c|c|}
\hline \multirow[t]{2}{*}{ Team } & & \multicolumn{6}{|c|}{ Toronto Raptors } \\
\hline & & $N=13$ & & $\mathrm{~N}=7$ & & $\mathrm{~N}=4$ & \\
\hline & & 1 to 9 & & 10 to 19 & & 20 and & \\
\hline & & Points & Min & Points & Min & Points & Min \\
\hline \multirow[t]{12}{*}{ Player } & $\mathrm{X} 1$ & 0 & 0 & 0 & 0 & 0 & 0 \\
\hline & X2 & 0 & 0 & 0 & 0 & 0 & 0 \\
\hline & X3 & 17.6 & 13.9 & 20.2 & 26.5 & 12.8 & 19.1 \\
\hline & $X 4$ & 18.6 & 6.5 & 4.4 & 2.3 & 10.2 & 10.9 \\
\hline & $\times 5$ & 10.5 & 14.8 & 19.1 & 23.4 & 27.7 & 21.5 \\
\hline & $\mathrm{X} 6$ & 23.3 & 20.5 & 21.7 & 14.9 & 28.1 & 13.6 \\
\hline & $\mathrm{X} 7$ & 35.7 & 24.9 & 34.1 & 33.3 & 12.5 & 21.7 \\
\hline & $\mathrm{X} 8$ & 28.7 & 19.2 & 19.7 & 17.2 & 14.7 & 12.2 \\
\hline & X9 & 22.5 & 18.4 & 24.3 & 13.2 & 32.1 & 21.3 \\
\hline & $\mathrm{X} 10$ & 31.4 & 29.6 & 27.8 & 22.2 & 26.6 & 27.4 \\
\hline & $\mathrm{X} 11$ & 0 & 0 & 0 & 0 & 0 & 0 \\
\hline & $\mathrm{X} 12$ & 0 & 0 & 0 & 0 & 0 & 0 \\
\hline
\end{tabular}

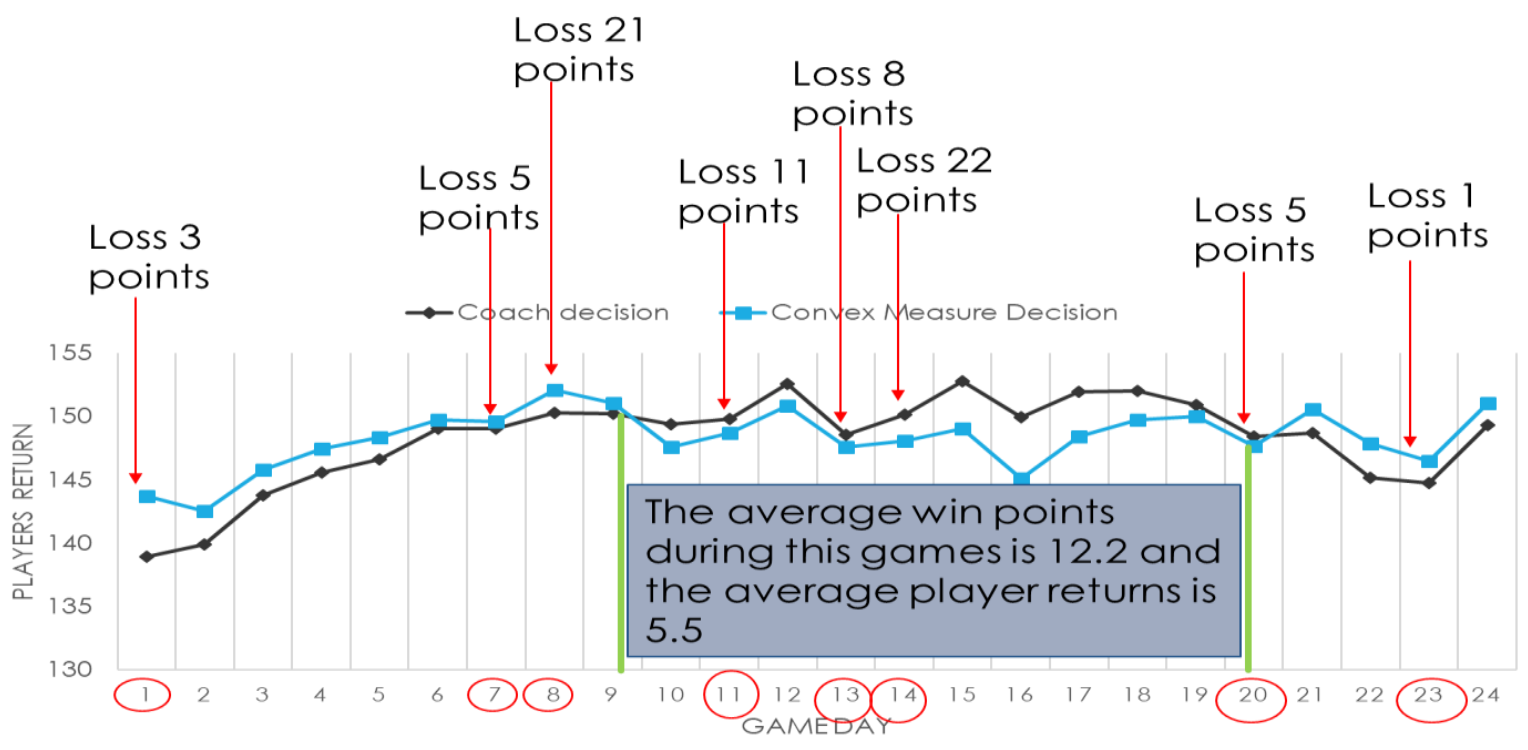

Figure 2. Two techniques over time.

The lost games were at period one, seven, eight, eleven, thirteen, fourteen, twenty and twenty-three on average of 9.4 points. In this period the optimal strategy is convex for game days; one, seven, eight and twenty-three. Furthermore, the losses come on average of 7.4 less than the total average, investigate that the optimal technique to win the games is the ARC program. In addition, over the period nine to twenty the average winning points during the game is 12.2 and the coach decision is the optimal. 


\section{CONCLUSION}

Concluded this research idea the following optimization problem under convex measures and investigate that the previews theorem has a perfect fit and over the basketball game. The ARC program is an action-based framework and a practical program to identify optimal athletes' submission and to correct random performance errors in top-level athletes. From the applied perspective, the ARC program was used to improve the quality of the coaching process and to enhance systematic work on sport techniques. Our findings suggest that: under the convex risk measures, coaches can optimize players returns. The support performance analyst team can run the following program to find the best player selection into different circumstances. We show that with the proposed method we are able to compare the optimal strategies, taking into account possible confounding factors such as shooting percentage, the offensive rebounds, the turnovers and the number of free throws. We provided a linear algorithm that summarizes the steps to achieve this useful ARC convex optimization problem.

\section{REFERENCES}

Artzner, P., Delbaen, F., Eber, J. M., \& Heath, D. (1999). Coherent measures of risk. Mathematical finance, 9(3), 203-228. https://doi.org/10.1111/1467-9965.00068

Boyd, S., Vandenberghe, L.: Convex Optimization. Cambridge University Press, 2003.

Chen, Z., \& Wang, Y. (2008). Two-sided coherent risk measures and their application in realistic portfolio optimization. Journal of Banking \& Finance, 32(12), 2667-2673. https://doi.org/10.1016/i.jbankfin.2008.07.004

Cheng, D., Berezovski, T., \& Sezen-Barrie, A. (2016). Aiming a Basketball for a Rebound: Student Solutions Using Dynamic Geometry Software. Illinois Mathematics Teacher, 63(1), 34-39.

Ibáñez, S. J., Sampaio, J., Feu, S., Lorenzo, A., Gómez, M. A., \& Ortega, E. (2008). Basketball gamerelated statistics that discriminate between teams' season-long success. European journal of sport science, 8(6), 369-372. https://doi.org/10.1080/17461390802261470

Kashan, A. H. (2009, December). League championship algorithm: a new algorithm for numerical function optimization. In 2009 International Conference of Soft Computing and Pattern Recognition (pp. 43-48). IEEE. https://doi.org/10.1109/socpar.2009.21

Lennartsson, J. (2014). Probabilistic modeling in sports, finance and weather.

Luenberger, D. G., \& Ye, Y. (1984). Linear and nonlinear programming (Vol. 2). Reading, MA: Addisonwesley.

Mendes, L., \& Janeira, M. (2001). Basketball performance-multivariate study in Portuguese professional male basketball teams. Notational Analysis of sport-IV, 103, 111.

Oliver, D. (2004). Basketball on paper: rules and tools for performance analysis. Potomac Books, Inc.

Sampaio, J., Janeira, M., Ibáñez, S., \& Lorenzo, A. (2006). Discriminant analysis of game-related statistics between basketball guards, forwards and centres in three professional leagues. European journal of sport science, 6(3), 173-178. https://doi.org/10.1080/17461390600676200

Tsamourtzis, E. (2002). Video analysis programmes in Coaching. Board of Trustees for the Evaluation and Management of the Property of the Democritus University of Thrace.

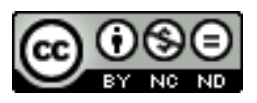

This work is licensed under a Attribution-NonCommercial-NoDerivatives 4.0 International (CC BY-NC-ND 4.0). 